\title{
A Coherent Subregion Time-Reversal Imaging Approach Using Temporal Filtering
}

\author{
Vahid Hassanzade ${ }^{1}$, Ahad Tavakoli ${ }^{1}$, and Gholamreza Moradi ${ }^{1}$ \\ ${ }^{1}$ Amirkabir University of Technology
}

August 7, 2020

\begin{abstract}
A Time-Reversal ( TR) imaging method for multiple targets that incorporates fewer transmitters is suggested using temporal filtering and Frequency Focusing (FF) matrices. This study is intended for the detection of malignant breast tumors with minimal microwave radiation. A couple of scenarios with two and four tumors are considered here. Using only two transmitters and twelve receivers, we show that two tumors could be detected in an inhomogeneous tissue breast. The results are compared with conventional TR Multiple Signal Classification method (MUSIC), which requires more transmitters. It is shown that several well-resolved tumors that exceed transmitters could be detected by subregion Spatio-Temporal Filtering (SSTF). In our proposed method, we used the orthogonal subspace instead of the signal subspace to have a higher Signal to Clutter Ratios (SCR). Since the Born approximation is not used, multiple scattering is considered. In addition, white Gaussian noise is added to measurement simulations. A 3-GHz modulated Gaussian pulse with a 1-GHz bandwidth is employed for illuminating the medium. Finite-Element Time-Domain method (FETD) is used in the measurement simulations, and the analytic Green's function of the background is utilized for backpropagation. Several simulations are provided that show the effectiveness of this approach.
\end{abstract}

\section{Hosted file}

ENGR_VH1.docx available at https://authorea.com/users/349395/articles/474534-a-coherentsubregion-time-reversal-imaging-approach-using-temporal-filtering 